\title{
Organizational factors and the perception of motion in depth
}

\author{
DONALD H. MERSHON, THOMAS A. JONES, and MARY E. TAYLOR \\ North Carolina State University, Raleigh, North Carolina
}

\begin{abstract}
When two stationary, stereoscopically separated targets are viewed in a completely dark surround, and no cues concerning their egocentric distances from the observer are salient, the farther target tends to be seen at the same distance it would have assumed if it were by itself. The nearer target is seen as being closer than it would have been if seen alone. The present studies extend this previous finding (now termed the far-anchor effect) into the domain of targets that move in stereoscopic space. Observers viewed two small illuminated targets, which began at either the same or different stereoscopic distances. One of the targets was moved in depth and the observers identified the target that appeared to move. Conditions varied according to the initial depth location of the moving target. Significantly more correct responses were reported when the nearer target moved than when the farther one moved, consistent with the hypothesis that the perception of motion in depth is affected by the aforementioned perceptual anchoring effect of the farther target.
\end{abstract}

Consider the factors that determine the apparent distance of a small, physically remote point of light observed binocularly in an otherwise completely dark surround. Few cues exist to mark its egocentric distance from the observer. At best, the observer will gain some information from the states of accommodation and vergence (Morrison \& Whiteside, 1984), both of which should indicate that the point is at a generally far distance. Now consider a second point of light, farther away in physical space. It too provides limited cues to its egocentric distance.

For each of the above targets, however, a perceptual organizational factor, termed the specific distance tendency may play a role (Gogel, 1969, 1977). Specifically, this is the tendency of observers to perceive any object as if it were located some specific distance away (typically, between 1 and $2 \mathrm{~m}$ ). Although considered to be an omnipresent factor in the perception of visual distance, the specific distance tendency usually has its greatest effect on reports of distance when few cues to distance are available (e.g., under reduced conditions of viewing). For each of the target points described above, the specific distance tendency would thus be expected to have a significant impact upon perceived distance. It would not, however, be the sole basis for the perception of distance. Gogel (1972) proposed that the apparent distance to any

The authors thank John Cook, Tim O'Brien, and William Swallow of the Department of Statistics, North Carolina State University, for statistical consultation on these studies. We also gratefully acknowledge the assistance of Sandy Kozar in the data collection and the helpful comments from David Clarke and from several anonymous reviewers. Requests for reprints should be sent to $D$. H. Mershon, Department of Psychology, Box 7801, North Carolina State University, Raleigh, NC 27695-7801. The current address of the second author is T. A. Jones, 1100 Astor Street, Norristown, PA 19401. such target would always represent a combined effect of the specific distance tendency and whatever residual (oculomotor) cues to distance that might exist. He termed this combined effect the egocentric reference distance. With the difference in residual egocentric cues intentionally minimized (as in our example), each individual target should appear near the same reference distance.

Now imagine the same two targets presented simultaneously. Due to the difference in the physical distances of the two targets, the relative cue of binocular disparity should allow observers to see that the targets are at clearly different distances. But where should they appear in egocentric space? Although several plausible alternatives might be suggested, Gogel's (1972) research makes it clear that the farther target consistently assumes the egocentric reference distance and the nearer target appears to be closer than that reference distance. Similar results were obtained by Foley (1985). Another study showed that fundamentally the same effect occurs, even when the nearer target is a larger (and perhaps perceptually more "stable") rectangle, rather than a small point of light (Mershon \& Lembo, 1977). Mershon, Granberry-Hager, Bartlett, \& DeCamp (1981) found that the effect also occurs when several additional lights are presented at depth locations between the nearest and farthest targets. In that study, the apparent distance of the entire configuration of lights was perceptually seen with the farthest lights remaining at essentially the same egocentric distance as they had been when viewed in isolation. The stability of the farthest target even seems to occur when the depth interval between near and far targets is defined by a collection of monocular cues, rather than by stereopsis (Mershon, Voncannon, \& Windes, 1976). Thus, the effect of interest seems to depend more upon the operation of an autochthonous organizational factor (i.e., the specific distance 
tendency) than upon the particular targets or the particular visual cues employed in a given situation. Overall, the phenomenon (hereinafter referred to as the far-anchor effect) appears to be both pervasive and robust.

The above studies suggest that certain errors in the perception of motion in depth may be more likely than others. Specifically, imagine a simple binocular configuration consisting of a single near and a single far target. Further imagine that the far target indeed assumes the egocentric reference distance. If the far target were moved in depth, but still remained the farthest target in the configuration perceptually, then it should continue to be seen as if it were at the egocentric reference distance. ${ }^{1}$ Under such conditions, changes in the stereoscopic depth interval that result from motion of the far target might be perceptually attributed to opposite movements of the nearer target. (This prediction arises from the expectation that the totality of relative changes within a display will tend to be perceptually distributed among the targets involved; see Gogel, 1984; Rock, Auster, Schiffman, \& Wheeler, 1980.) Thus, the perceptual tendency to position an egocentrically ambiguous depth interval in distance, on the basis of the far-anchor effect, should induce the illusion of movement in the "less stable" nearer target. Examining this possibility was the main concern of the present experiments.

\section{Other Considerations for the Present Studies}

Presenting an observer with motion in depth entails several variables. Direction of motion, for example, may be either toward or away from the observer. Also, the distance a target moves and the time to cover that distance (speed) are inherent factors in motion in depth. Following are some of the considerations involved in the choices made for the present investigations.

Several studies have indicated that human beings may respond differently to movement that involves an approaching target than to movement that involves a receding one (Ball \& Sekuler, 1980; Perrone, 1986). It has been suggested that such differences arise from the greater experience with expanding patterns of optical flow (such as those that occur naturally during forward locomotion). Since we do not customarily move around with our eyes directed to the back, we have not developed sophisticated processes for dealing with contracting patterns of flow.

In a simpler display that had more similarities to our own, Janssen, Michon, and Harvey (1976) found that forward motion was perceived significantly more readily than was receding motion. However, their study involved judgments of absolute motion and a target moving in an otherwise dark surround. No additional objects in the display offered any reference by which to judge the target's motion. This difference may not persist in cases of relative motion, in which the observer has another visual reference. Indeed, unless there is a reference mark of some sort, there may be no sense of motion per se, even when changes in vergence occur (Erkelens \& Regan, 1986). In the present research, the use of displays with one moving and one stationary target ensured that a suitable reference mark was always available.

Hong and Regan (1989) found that many individuals may be differentially blind to one direction of motion in depth, even though they may be able to correctly report the opposite motion (e.g., approaching vs. receding motions). The demonstration of such field defects, however, was accomplished by the use of very short presentations $(<1 \mathrm{sec})$. Longer presentations of the moving targets allow an observer the opportunity to conver the display from crossed to uncrossed disparities, or the reverse. Therefore, to avoid problems with this sort of field defect, the observers in the present studies were not constrained as to fixation, and observation periods were long enough (at least $5 \mathrm{sec}$ ) to allow use of the preferred fixation pattern. Such free viewing conditions also had the advantage of being more similar to the tasks involved in daily living.

The factor of speed was also of concern. A target moving too quickly may introduce a relatively abrupt change in its vergence angle, providing a strong (if transient) cue for a change in egocentric distance that would otherwise be absent. Although even large and rapid changes in vergence do not seem to contribute directly to a motion-indepth mechanism (Regan, Erkelens, \& Collewijn, 1986), such an abrupt change in the absolute "position in depth" could provide an artifactual indicator that one, rather than the other, target had moved. Such extraneous information could then influence reports of the perceptual effects expected from the egocentric organizational process. In order to evaluate the effects of speed, the targets in the different conditions of Experiment 2 covered the same depth extent in different durations.

\section{GENERAL METHOD}

Two small ( $12.7 \mathrm{~cm}$ diagonal) Panasonic Video monitors (controlled by a Commodore 64 computer), some half-silvered mirrors, and crossed-polarizing filters were used to create a polaroid stereoscope. The monitors always remained at a physical distance of approximately $4 \mathrm{~m}$ from the observer. Perceptually distinct images of two white rectangles of equal size (approximately $4 \mathrm{~mm}$ wide $\times 6 \mathrm{~mm}$ high) were presented using this stereoscope. From the normal position of the observer, these target rectangles were $3.4^{\prime} \times$ $5.2^{\prime}$ of arc in size and were nominally $0.65^{\circ}$ apart laterally (center to center). Depending upon the lateral positions of the component images on the screens, these targets could be stereoscopically separated in depth by as much as $23^{\prime}$ of arc. Thus, either target could assume equivalent vergence distances anywhere between 2.85 and $4.05 \mathrm{~m}$ from the observer. Direct measurement of target luminances was not feasible, but calculation indicated that effective luminances at the eye were approximately $0.03 \mathrm{~cd} / \mathrm{m}^{2}$.

When desired, lateral movement of the screen images created the perceptual effect of motion in depth without changing the size or brightness of either target. Two speeds of movement were available by introducing different software-controlled delays whenever the images shifted by 1 pixel on the screen. At the slower speed, the moving target required $10.8 \mathrm{sec}$ to cover the simulated $1.2-\mathrm{m}$ 
separation in depth. The same separation was covered in $6.5 \mathrm{sec}$ at the faster rate. In all trials in which one of the targets moved in depth, the motion continued until that target had reached the other extreme of distance. Both targets then disappeared.

Because the lateral motion of the images was constant on the screen (within each condition), the stereoscopic motion in depth was not. Unavoidably, the vergence distance of the moving target changed more rapidly as vergence increased. For the slower speed, the equivalent rate of motion in depth varied between $8.0 \mathrm{~cm} / \mathrm{sec}$ and $15.4 \mathrm{~cm} / \mathrm{sec}$. For the faster speed, the rate varied between $13.3 \mathrm{~cm} / \mathrm{sec}$ and $25.6 \mathrm{~cm} / \mathrm{sec}$. Perhaps because of the relatively short duration of observation and the smoothly continuous change in rate, no noticeable variations in speed were apparent for either direction of motion. In any case, effects of such accelerations/decelerations were controlled for by the design of Experiment 3. In that experiment, the exact same physical displacement was paired on separate presentations with both a nearer and a farther target. Thus, any effect of changes in the rate of 3-D motion were balanced across conditions with opposite predictions.

The rectangular targets were presented in an otherwise completely darkened visual alley. The observers viewed the display through an aperture from a darkened booth. A shutter in front of the aperture prevented any view of the alley before or after an experimental presentation. The observer and experimenter communicated via an intercom system.

A special effort was made to circumvent a problem with binocular fusion, which may occur in a severely reduced viewing environment. With only the two small targets visible and no other framework to support stable fusion, there was a tendency for some of the observers to see three or even four lights. (The observers who were unable to fuse the left- and right-eye views within a minute or so of the start of a presentation were replaced.) $)^{2}$ To minimize initial fusional difficulties, as well as subsequent problems with the fused display breaking up, the depth interval used in the present studies was kept fairly small. Although both expanding and contracting depth intervals were examined, no interval exceeded the $1.2 \mathrm{~m}\left(23^{\prime}\right.$ of arc) maximum depth separation.

\section{EXPERIMENT 1}

\section{Method}

Observers. A total of 60 observers participated in Experiment 1. Half of the observers in any condition were men and half were women. The observers were drawn from a pool of introductory psychology students who participated as one means of fulfilling a course requirement. Each observer had a visual acuity, corrected if necessary, of at least 20/30 in both eyes and a stereoacuity of at least $145^{\prime \prime}$ of arc. The observers were unfamiliar with the laboratory and the purpose of the experiment.

Procedure. Each observer first took an acuity test using a standard Snellen wall chart and a stereoacuity test using the Keystone Multi-Stereo Test. A measure of interpupillary separation was also taken, in order to calibrate the polaroid stereoscope individually, so that the targets appeared at the same stereoscopic distances for each observer. The observer was then seated in the observation booth and was allowed $1 \mathrm{~min}$ to adapt to the dark. Each observer was presented with the appropriate display and was asked to report the apparent distance between him/herself and the visual target(s) in "feet, inches, or some combination of feet and inches."

Experiment 1 was conducted as a replication of previous work with simple binocular configurations (Gogel, 1972; Mershon \& Lembo, 1977). It was designed merely to confirm that the present equipment and procedures would demonstrate the same general stability in the perceived distance to the far target (and the same change in the perceived distance to the near target) as previous research. For Experiment 1, therefore, all the targets remained stationary throughout a presentation. Twenty of the 60 observers were presented with the near target alone, 20 were presented with the far target alone, and 20 were presented with the near and far targets simultaneously. These three conditions were presented in randomly ordered blocks of 3 observers each. The observers were run in the order in which they reported to the laboratory.

Rationale and predictions. In Experiment 1, each target individually should assume an apparent distance determined by the egocentric reference distance. In addition, the perceived distance to the binocularly farther target should not change significantly when it is viewed in combination with the nearer target. However, the perceived distance to the nearer target presented in combination with the farther target should be seen as significantly closer than when presented alone. These findings would be consistent with previous studies on the perceptual positioning of an egocentrically ambiguous binocular interval. They would further verify the existence of the perceptual conditions that were necessary for testing the main hypothesis concerning perceived motion in depth.

\section{Results}

Table 1 summarizes the results for the three groups of observers in Experiment 1. (Medians, rather than mean values, are given because of the tendency for verbal reports of apparent distance to be highly skewed; Mershon \& Lembo, 1977.) A Mann-Whitney $U$ test, comparing the responses for the near and far targets presented individually, yielded a nonsignificant $U$ value of 191 ( $p>$ .05 ). Thus, there is no evidence that the judgments for the individual targets were different. Indeed, the median report of perceived distance to the nearer target was slightly greater than the median report of perceived distance for the farther target.

A second Mann-Whitney $U$ test was used to compare the reports of the perceived distance of the far target presented alone with judgments of the far target presented in conjunction with the near one. This yielded a nonsignificant value of $193(p>.05)$. Thus, the presence or

Table 1

Verbal Reports of Perceived Distance in Experiments 1 and 3

\begin{tabular}{|c|c|c|c|c|c|c|}
\hline & \multirow{2}{*}{\multicolumn{2}{|c|}{$\frac{\text { Individual Lights }}{\text { Experiment } 1}$}} & \multicolumn{4}{|c|}{ Simultaneous Lights } \\
\hline & & & \multicolumn{2}{|c|}{ Experiment 1} & \multicolumn{2}{|c|}{ Experiment 3} \\
\hline & Near & Far & Near & Far & Near & Far \\
\hline$\overline{\mathrm{Med}}$ & 1.52 & 1.22 & 0.91 & 1.37 & 1.17 & 1.37 \\
\hline Semiinterquar & 0.46 & 1.14 & 0.36 & 0.46 & 0.61 & 1.09 \\
\hline Geometrical means & 1.41 & 1.57 & 0.93 & 1.39 & 1.16 & 1.43 \\
\hline
\end{tabular}

Note-All values converted to meters. 
absence of a near target did not reliably affect the observers' distance judgments to the farther target. A third $U$ test, comparing the perceived distance of the near target presented alone with judgments of the near target presented with the far one, however, yielded a value of 135 . This value was statistically significant $(p<.05$, onetailed). Thus, the observers' distance judgments to the near target were significantly closer when presented in conjunction with a farther target, whereas distance judgments to the far target itself were not significantly changed. ${ }^{3}$ The current display was deemed capable of serving in Experiment 2.

In Experiment 1, male and female observers and left versus right sides of the display were counterbalanced. Neither of these variables, however, was associated with any significant differences.

\section{EXPERIMENT 2}

\section{Method}

Observers. Another (different) 320 observers from the same source and with the same characteristics as those in Experiment 1 served in Experiment 2. Again, half of the observers in any given condition were men and half were women.

Procedure. Following preliminary instructions and a brief period of dark adaptation, each observer viewed a display of two illuminated targets. For half of the observers, this display consisted of a near and a far target. The independent variables were whether the near or the far target was moved, whether the moving target was on the left or right side of the display, and whether the speed of movement was fast or slow. The other half of the observers saw a display with two targets at the same distance-either two far targets or two near targets. For these observers, the independent variables were the initial distance of the pair of targets, whether the moving target was on the left or the right side of the display, and whether the speed of movement was fast or slow. This second group of observers was intended as a control condition, because one might expect a priori that correct and incorrect judgments should be 50-50 when there was no initial depth separation (given the limited viewing conditions).

Each observer was asked to report the distance to each target (primarily to confirm that a clear depth separation was seen in the near-far simultaneous condition). Each observer was then presented with a single trial in which one of the targets in the pair was moved, and was asked to report whether the near or the far target had moved in depth and the direction of that movement.

There were 32 conditions in all, including the division of observers by sex. Each condition was presented to 10 different observers. The observers and conditions were run in a randomized order.

Rationale and predictions. In Experiment 2, we tested the hypothesis that the relative position of a target within a binocular depth configuration would have a differential effect on the perceived motion in depth. Specifically, the far-anchor effect was expected to result in any stereoscopic variation being seen as motion of the nearer target. (Figure 1 clarifies the primary display conditions and these expectations.) Thus, we expected more correct reports when the near target actually moved back and more errors in the motion reports when the far target moved forward. For the control conditions, in which both targets started at the same distance, no difference in performance was expected (but see the Discussion section for Experiment 2).

\section{Results}

Each observer was presented with either a receding near target or an approaching far target. Verbal reports as to

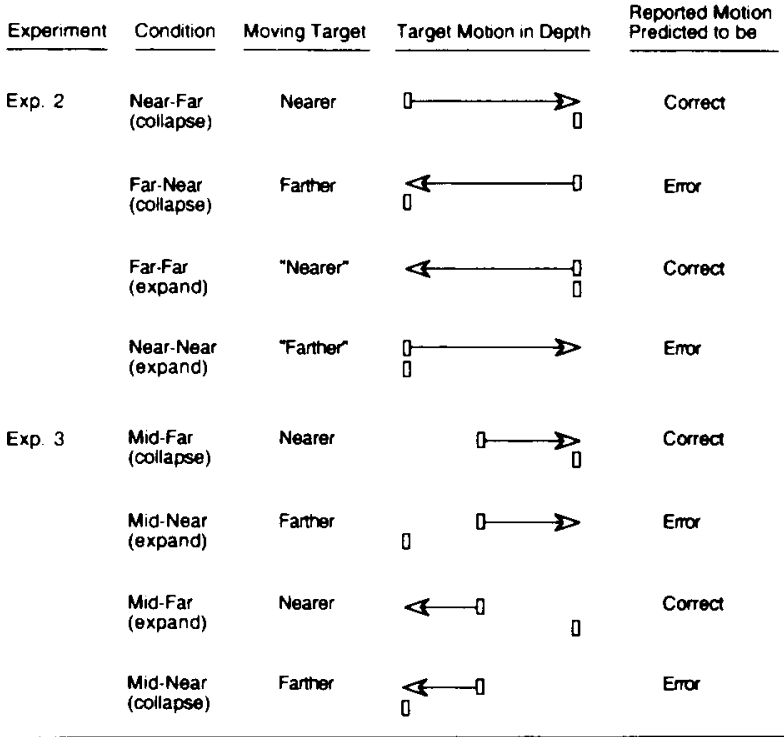

Figure 1. The primary conditions used in Experiments 2 and 3, including top views of how the targets moved in depth and the expected performance. For simplicity, the left-hand target is always shown as the moving target. For Experiment 2, the lateral position of the moving target was balanced across observers. For Experiment 3, the lateral position of the moving target was balanced across presentations to each observer. (The assumed, but not shown, location of the observer is to the left of the figure.)

which target appeared to move and in which direction were categorized as correct if the observer's response exactly matched the veridical physical motion, and incorrect otherwise. In the instances in which an observer gave a first impression and then immediately changed it, the second impression was the response that was categorized. (The second impression was chosen, because the observers generally reported that their second impression was clearer or was the one that lasted for the greater duration of time while they were watching the display.)

Logistic regression. Logistic regression (Aldrich \& Nelson, 1984) was used to analyze correct versus incorrect responses. Like linear regression, logistic regression is used to construct a model that describes how the value of a dependent variable changes with the value(s) of some independent variable(s) or with interactions of those variables. However, logistic regression is used when the dependent variable is categorical. More specifically, the dependent variable is a probability that can take on only one of two possible values (commonly represented as either 0 or 1 ). Linear regression is inappropriate in this case because fitting a linear model to measures confined to just two given points ( 0 and 1$)$ grossly violates the assumption that the dependent variable may take on an infinite set of values. The logistic regression procedure first requires that the frequency of "positive" outcomes be determined for each cell in the overall design. These cell frequencies are then transformed into log values, to create a continuous variable. The end result of a logistic regression is a set of the independent variables (a model) 
that is significant in determining the probability of a positive response for the dependent variable.

In this study, logistic regression tests were used to determine the probabilities of being able to predict correct versus incorrect responses. The analyses constructed backward stepwise models until only the independent variables or interactions that were significant as predictors were left. A separate test was conducted on the data from the conditions with one near and one far target and on the data from the conditions in which both targets were initially at the same distance.

Goodness of fit for model analyses. Overall, the two regression models constructed by the above analyses were fairly successful in "predicting" the correct percentages of the responses. For the analysis of the near-far and far-near conditions, the model correctly predicted $69.8 \%$ of the responses $(r=.323)$. For the analysis of the farfar and near-near conditions, the model correctly predicted $67.2 \%$ of the responses $(r=.296)$. Note that although the $r$ values for each of these models may be used for evaluating the models in a "goodness of fit" type of comparison, it is important to understand that the $r$ and $r^{2}$ values found in a standard regression analysis are different from the $r$ values reported for logistic regression (Aldrich \& Nelson, 1984). Briefly, this is because in a standard regression analysis, the $r$ value is based on a mean and a variance of each dependent variable. With categorical data, estimating a proportion in lieu of the variance is appropriate. The $r$ value reported here, therefore, is determined by comparing the predicted values with the actual responses in the final model. (Predicted values are attained by using the logistic regression formula to determine whether the probability of getting a positive result is greater than or less. than .5 for each cell.)

Primary analyses. Table 2 lists significant variables for each of the primary logistic regression analyses, including response frequencies and the chi-square values, the $p$ values, and the $r$ values.

The first logistic regression analysis compared responses for the presentations that included both a near and a far target. Two variables were found to be significant at the .05 level in constructing a regression model: target in motion (whether the moving target was near or far; $p<.0001$ ) and lateral position of the near target (whether the near target was on the left or right side of the display; $p=.0189$ ). The statistical significance of the target-inmotion variable supports the hypothesized influence of the far-anchor effect on the perception of motion in depth in an egocentrically ambiguous display. The unpredicted finding of a significant lateral position variable indicates a modest anisotropy; examination of the detailed results shows that this observed asymmetry reflects improved performance on the presentations in which the near target was on the right and was actually in motion. Within each condition of lateral position, however, the primary (predicted) finding of better performance when the near target moved held consistently $(45.0 \%$ correct on near, $22.5 \%$ correct on far, when the near target was on the left; $72.5 \%$ correct on near, $30.0 \%$ correct on far, when the near target was on the right).

The second logistic regression analysis compared conditions in which the two targets started at the same distance. Only the variable of configuration (whether the configuration was far-far or near-near; $p=.0002$ ) was found to be significant. The significance of this variable seems at first to be a surprise, because the frequencies of correct reports are not equal. Perhaps this finding may be predictable from the results of Janssen, Michon, and Harvey (1976). As noted earlier, those researchers found that forward motion was reported more readily than receding motion. In Experiment 2, when one of the near-near targets moved back, erroneous forward motion in depth tended to be seen for its mate. This suggests that it may have been easier to see a target moving forward, even when such an outcome was incorrect. This interpretation, however, is inconsistent with the results of the near-far conditions, because in those situations the nearer target was more often seen to move, even though it appeared to recede. Therefore, a somewhat different interpretation will be discussed below.

Secondary analyses. As a confirmation procedure, additional logistic regression analyses were conducted on a modified data set. For these analyses, incorrect responses were categorized into responses that were "possible," given the stereoscopic changes presented, and responses that were "inconsistent" with any predictable perceptual

Table 2

Significant Variables for Analyses of Configurations in

Experiment 2, Showing Number and Percent of Correct Responses, With Other Values Showing Results of the Logistic Regression Analyses

\begin{tabular}{|c|c|c|c|c|c|c|}
\hline Variable & Level & No. Correct & Percent Correct & $x^{2}$ & $p$ & $r$ \\
\hline \multicolumn{7}{|c|}{ Far-Near and Near-Far Configurations } \\
\hline Target-in-motion & $\begin{array}{l}\text { Near } \\
\text { Far }\end{array}$ & $\begin{array}{l}47 \\
21\end{array}$ & $\begin{array}{l}58.8 \\
26.2\end{array}$ & 16.96 & $<.0001$ & .262 \\
\hline Lateral position & $\begin{array}{l}\text { Near on Left } \\
\text { Near on Right }\end{array}$ & $\begin{array}{l}27 \\
41\end{array}$ & $\begin{array}{l}33.8 \\
51.2\end{array}$ & 5.51 & .0189 & -.127 \\
\hline \multicolumn{7}{|c|}{ Far-Far and Near-Near Configurations } \\
\hline Configuration & $\begin{array}{l}\text { Far-Far } \\
\text { Near-Near }\end{array}$ & $\begin{array}{l}45 \\
21\end{array}$ & $\begin{array}{l}56.2 \\
26.2\end{array}$ & 14.30 & .0002 & .238 \\
\hline
\end{tabular}


outcome (e.g., reporting a depth interval that expanded, rather than collapsed, in the conditions starting with a near and a far target). The incorrect responses expected from the far-anchor effect included only the responses that represented incorrect, but possible, alternatives (i.e., errors that reflected the induction of an opposite motion in a physically stationary target). Inconsistent errors accounted for 53 of the 320 total observations. A reduced data set was created by the elimination of such errors. Removing these observers did not noticeably affect the overall proportions of correct/incorrect judgments for either near or far targets. Logistic regression analyses conducted on the modified data set served to confirm the major findings of the primary analyses-that the depth location of the moving target is a significant determiner of performance ( $p=.0001$ for both the near-far conditions and the conditions starting with the targets at the same distance).

In addition, the new analyses showed an effect of the speed variable (with the faster speed producing a marginally greater number of correct responses in the nearfar conditions; $p=.0049$ ). There were also some irregular, two-way interactions of the lateral position variables by speed in the near-far conditions and lateral position $x$ sex of the observer in the same-distance conditions. More specifically, in the near-far conditions, the differences due to speed only occurred clearly (1) when the near target was on the left, or (2) when the moving target was on the left. In the near-near versus far-far analysis, the interaction may be interpreted as showing a slightly greater performance difference (men $>$ women) for presentations in which the moving target was located on the right than when the moving target was located on the left. Although no immediate explanations for these interactions are obvious, they do not have a major impact on the overall interpretation of Experiment 2. Rather, they may be considered as details that should be controlled or examined more thoroughly in Experiment 3.

\section{Discussion}

Near-far conditions. The significantly reduced frequency of correct responses reported when the far target moved than when the near one moved supports the primary hypothesis of this study. That is, the specific distance tendency appears to have an anchoring effect on the farthest target in a display. Thus, the far target is more apt to be perceived as stationary, even when it is moving in depth. Motion in depth of the near target is more likely to be perceived correctly.

Although they only occurred for the analyses with the modified data (after removal of the inconsistent errors), the possible contributions of target speed deserve comment. In particular, the greater frequency of correct responses sometimes reported when a target moved quickly supports the notion that the presence of a sudden and rapid change in vergence may disrupt the required conditions of egocentric ambiguity. The main effect of speed, in addition to the two interactions with the speed variable, indicates that the use of slower changes in depth may be better suited for testing the current hypothesis.
Far-far condition compared with near-near condition. In the analysis of responses for the far-far and near-near conditions, configuration was significant. Responses for both these display configurations were originally expected to be about $50 \%$ correct, because no clear far-anchor effect could be predicted when the display configuration did not have a distinguishably farther point. However, in both of these conditions, once the motion began, the configuration quickly became a near-far configuration. For example, the far-far configuration essentially became a near-far configuration, in which the moving "nearer" target continued to advance toward the observer. Likewise, the near-near configuration could be described as a near-far configuration, in which the moving "farther" target continued to recede away from the observer. Thus, these two configurations could be considered as near-far configurations with an added temporal, or time delay, variable. This being the case, we would then expect that for the far-far condition, in which the eventual "near" target was always the moving target, more correct responses would be reported than in the near-near condition in which the eventual "far" target moved. That is, as soon as either target moved enough so that an observer perceived a configuration with targets at different distances, the far-anchor effect would have begun to operate. Considering that there were $56.2 \%$ correct responses in the far-far condition ("near" target moving) and only $26.2 \%$ correct responses in the near-near condition ("far" target moving), an unpredicted anchoring effect may have occurred in much the same way as in the configurations that were initially near-far.

If the preceding interpretation is correct, it also provides more generality for the experimental findings. Although we intentionally avoided expansion beyond the $1.2-\mathrm{m} \mathrm{ex}$ tent used in our near-far conditions, the results from the two same-distance conditions indicate that expanding, as well as contracting, depth intervals may be affected. Thus, the stability of the farthest target may be an important factor as soon as a depth interval of any sort is created from an equidistant display.

Concerns and implications. The results of Experiment 2 suggest that an organizational factor, termed the far-anchor effect, can influence observers' ability to correctly discriminate stereoscopic motion in depth. As with most studies, this experiment leads to more questions. One concern involves the several observers who were unable to maintain adequate fusion to participate in the study. Quite possibly, those with fusional difficulties were those with very close values of tonic vergence (Owens \& Leibowitz, 1980). Such individuals would have had more trouble in binocularly fusing the extremely reduced display at the 4-m distance. A more structured display might help observers perceive the initial display correctly, as well as allowing an evaluation of the robustness of the faranchor effect through the introduction of more visual cues for the actual movements.

Another concern is the possibility that the observed results are primarily limited to collapsing depth intervals, 
despite the evidence from the far-far and near-near displays. Would the same effect be found if a preexisting depth interval were expanded? Also, although there were good reasons to use independent observers in Experiment 2 (to avoid the development of any possible response bias), it would be useful to know whether the far-anchor effect would continue throughout several judgments of motion in depth. That is, how stable is the effect over time? Such questions were the basis for undertaking Experiment 3.

\section{EXPERIMENT 3}

\section{Method}

Observers. Another 48 observers ( 24 men; 24 women) from the same source and with the same characteristics as those in Experiments 1 and 2 served in Experiment 3.

Apparatus. The visual displays used in Experiment 3 were developed from those of Experiment 2. Specifically, the starting position of one target in each of the previous displays was altered, so that one target began at an extreme distance (either 2.85 or $4.05 \mathrm{~m}$ ) and the other began at a distance of $3.35 \mathrm{~m}$. This position represented a midway location with respect to the images on the 2-D monitor screens and was chosen (rather than a true-depth midpoint) in order to control for the duration of exposure to the movement. The same starting positions in relative depth occurred equally often for all four cases of depth motion: (1) the "midpoint" target moved toward a far target (collapsing the depth interval); (2) the "midpoint" target moved away from a near target (thereby expanding the depth interval); (3) the "midpoint" target moved forward, away from a far target (expanding the depth interval); and (4) the "midpoint" target moved forward, toward a near target (collapsing the depth interval). Thus, the same physical target motion was presented equally often with a nearer target and a farther target (see Figure 1). In the first situation, the correct response and the predicted response agreed; in the second situation, the correct and predicted responses differed.

For Experiment 3, only the slower speed of motion was used. On the basis of Experiment 2, this speed was believed to be more likely to avoid artifactual egocentric changes and, given the decreased depth motions involved in the new displays, provided a better (longer) opportunity for observation. The exposure duration of the stereoscopic motion was thus approximately $5 \mathrm{sec}$ for each display.

Procedure. Following visual screening, preliminary instructions, and a brief period of dark adaptation, each observer viewed several displays, each consisting of two illuminated targets. These displays always began with one target located nearer to the observer than the other, with their depth separation approximately half that used for Experiment 2. The independent variables were whether the nearer or the farther target was moved, whether the moving target was on the left or right side of the display, and whether the initial interval expanded or collapsed in depth during the observation.

Each observer was shown a sample display and was given further instructions about the experiment. Half of the observers viewed a sample in which the left target was nearer and moved still closer to the observer (thereby expanding the depth interval); half of the observers viewed a sample in which the farther target was on the left and moved closer to the observer (collapsing the depth interval). The initial sample presentation was used solely to ensure that the observers would be able to see the depth interval clearly and understood the nature of the motion judgment task. The observers were also instructed to report any occasions in which fusion was lost before or during a trial (i.e., any occasions in which they saw more than the two intended targets).

Following the sample presentation, the shutter was closed and the first of 16 experimental displays was generated. When the shutter was reopened, the observer again saw two illuminated targets and was asked whether they appeared at the same or at different distances. If "different," the observer was asked to indicate which target appeared closer to him/herself. If these responses were correct, the observer was told that the motion was about to start and the trial itself was initiated. After the targets disappeared at the end of the movement, the observer reported whether the left or the right target had appeared to move. There was no effort to inquire about the direction of motion in depth, or to ask specifically whether the nearer or farther target had been moving, because of concern with introducing a bias over the course of the experiment. (For example, if the predicted far-anchor effect were important, then a majority of responses would have indicated the nearer target to be moving, regardless of the actual motion, resulting in possible response biases. On the other hand, equal numbers of left and right responses would tend to occur for any degree of the far-anchor effect.) At the end of the presentation, the shutter was again closed while the next display was readied. The remaining 15 trials continued in the same pattern. The intertrial interval was approximately $20-30 \mathrm{sec}$.

The first eight trials were divided equally between those with the nearer target on the left and those with the nearer target on the right, between those in which the nearer target moved and those in which the farther target moved, and between those in which the motion resulted in an expansion of the initial depth interval and those in which the motion resulted in a collapse of the initial depth interval. The order of the trials was random. A second set of trials included another presentation of each of these same eight possible conditions, in a different random order. Thus, overall, there were eight trials for which the target predicted to be seen as moving (by the far-anchor effect) was also the target actually in motion. For another eight trials, the predicted target and the correct target were different.

At the end of the experiment, the sample display was again presented. This time the observer was asked to report the apparent distance of each of the targets from him/herself in feet and/or inches. This judgment concluded the experiment.

\section{Results}

The main data from Experiment 3 consist of the number of trials (out of 16) in which the observer reported that the correct target was in motion and the number of trials (out of 16) in which the observer reported that the target in apparent motion was the one predicted by the far-anchor effect. It is clear from the experimental design that these numbers are partially dependent upon each other. Thus, if an observer were correct on either 0 or 16 trials, then the only possible value for "predicted score" would be 8 . If the correct score were 1 or 15 trials, then the predicted score could have varied between 7 and 9. If the correct score were exactly 8 , then the predicted score could have assumed any value between 0 and 16 . Figure 2 shows the paired scores for all the observers in Experiment 3. (The diamond-shaped area represents the region of possible pairings. If no systematic influences were present, then the data points should be distributed randomly throughout the diamond region. Systematic influences, on the other hand, would cause the data points to be displaced to the left or right of the vertical line and/or above or below the horizontal line.) Note the strong tendency for data points to be located to the right of center, indicating that predicted scores were higher than expected from chance. Note also that the correct scores tended to be distributed more or less evenly in the vertical domain. Eleven of the $\mathbf{4 8}$ observers had equal scores (correct vs. 


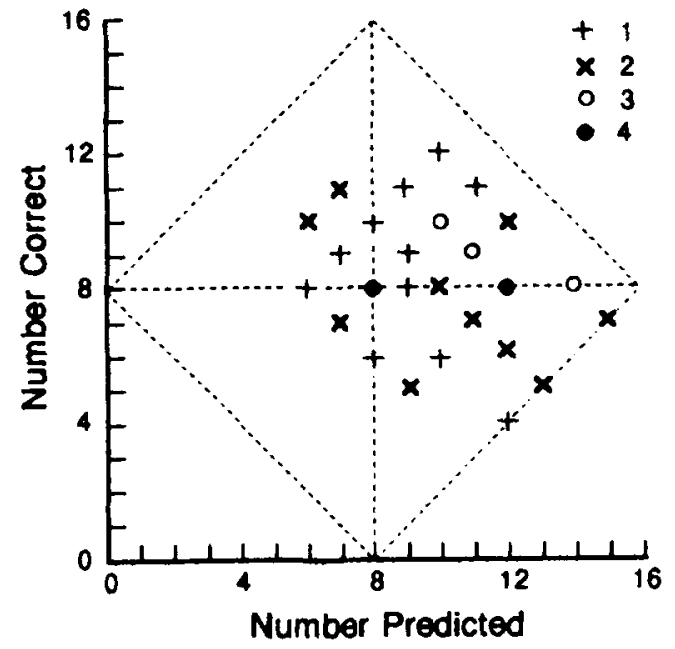

Figure 2. The data for the 48 observers in Experiment 3, shown as paired values of the number of correct responses versus the number of responses that were predicted by the far-anchor effect. The dashed diamond-shaped area defines the region of possible values. Note that the distribution of observers is more or less symmetrical around chance $(=8)$ when accuracy alone is considered. With regard to the predictions based on the far-anchor effect, however, observers clearly fall to the right of chance $(=8)$, indicating the effectiveness of this organizational concept in predicting the direction of perceived motion in depth. Four different symbols are used to indicate data points representing multiple observers.

predicted). Of the 37 other observers, 28 had predicted scores that were greater than their correct scores; only 9 had predicted scores that were less than their correct scores. A sign test showed this difference to be significant $(z=2.96, p=.003$, two-tailed). Furthermore, when one examines the trials for which a correct report of motion was given, one discovers that approximately two thirds of such reports occurred on trials for which the predicted response was the same as the correct response. This observation allowed a confirmatory test. A one-sample $t$ test on the proportions of correct responses that occurred on these "consistent" trials (against the null hypothesis that exactly half of the correct responses should have occurred by chance on consistent trials) indicated that the far-anchor effect may have been responsible for many of the correct responses $(t=6.00, p=.0001$, twotailed), as well as resulting in the predictable errors for other conditions.

No other significant motion effects were found in Experiment 3 . Thus, there were no differences attributable to the sex of the observer, no differences related to the lateral position of the near or far targets, no changes in the results as a function of comparing the first and second block of eight trials, and no interactions. There was no general tendency for the observers to favor either "left" or "right" as a response. When scores were compared with individual measures of stereoacuity, there was no indication that better or poorer stereoacuity (within the constraint that all the observers were required to discriminate $145^{\prime \prime}$ of arc before participating) was associated in any way with performance. Overall, the only significant effect was a clear ability of the far-anchor effect to predict observers' responses better than the assumption that they would tend to be correct and better than the assumption that scores would be distributed around chance.

Finally, the verbal reports of perceived distance $\left(D^{\prime}\right)$ obtained at the end of Experiment 3 were consistent with expectations. Not only were the perceived depth intervals approximately half the values found in Experiment 1 (consistent with the physical decrease in the average stereoscopic separation used), but $D^{\prime}$ for the far target was approximately the same as in Experiment 1, whereas $D^{\prime}$ for the near target was slightly greater than in Experiment 1 . Such a change is appropriate, because the faranchor effect should have kept the farther target relatively constant. The decrease in the average disparity used for the depth interval, therefore, should have resulted in a greater perceived distance for the perceptually more malleable near target. A comparison of the reported depth intervals of the $\mathbf{4 8}$ observers in Experiment 3 versus the reported depth intervals of the 20 observers in the simultaneous condition of Experiment 1 showed that the smaller stereoscopic interval of Experiment 3 resulted in a smaller reported interval (see Table 1). A one-way analysis of variance using ranked values (Conover and Iman, 1979) confirmed this observation $[F(1,66)=12.34, p=.0008]$. There was no point in conducting a statistical test on the near versus far targets of Experiment 3 itself, because the observers were replaced if they did not report the appropriate arrangement of the targets in depth.

\section{GENERAL DISCUSSION}

An interesting aspect of the present studies concerns the distinction that has been made between the mechanisms for static stereoscopic depth perception and stereoscopic motion in depth (Hong \& Regan, 1989). Our predictions concerned errors of perceived motion in depth; yet, the basis for these predictions (the far-anchor effect) arose from previous data gathered in a situation that involved the perception of static distances. This apparent contradiction may be resolved by the realization that the primary phenomenon involved here is probably not a stereoscopic effect at all. Rather, it appears to have more to do with the question of how scalar perceptual values become attached to exocentric intervals (see Gogel, 1977). In fact, one might speculate from the results with monocular depth intervals (Mershon et al., 1976) that movement of any "farthest"' target, whether separated from other targets by binocular cues or otherwise, should result in the perception of induced motion in depth of the nearer targets.

In different terms, the present studies do not really directly address issues in the perception of structure from motion or stereopsis (Lappin, 1990). Instead, they are concerned with the consequences of the operation of perceptual factors that add egocentric scale to relationships specified by any and all exocentric cues. They are also concerned with the establishment of the "scale factor" at which many investigations of structure from motion 
seem to stop. They may, nevertheless, have consequences for certain experimental situations used in those investigations in that they indicate a preferential position for the farthest targets in a display, at least with respect to certain types of judgments.

\section{Foley's Theory of Binocular Distance Perception}

It is important to note that the data obtained in the present studies are wholly consistent with those collected in research discussed previously by Foley $(1980,1985)$. In fact, his theory of binocular distance perception may be used to reach the same predictions as those based upon the specific distance tendency. To summarize, Foley proposed that the egocentric perception of any binocular configuration is characterized by a particular reference value of binocular parallax. (Binocular parallax would be equivalent to vergence distance, if fixation disparity were negligible; it is essentially the convergence "required" for a given binocular target.) When fixation is not restricted, this reference value is a weighted average of the parallaxes of the available targets, but with the greatest weight being given to the farthest target in the display. A measure of effective binocular parallax is linearly related to the reference binocular parallax and corresponds to customary measures of perceived egocentric distance. The position of any target in the display that is stereoscopically different from the reference parallax is determined jointly by the effective binocular parallax and the effective horizontal disparity between the target and that point.

In his 1985 paper, Foley presented data that show that variations in effective disparity produce changes in the perceived distance of a target, as long as that target is the closest one in a configuration. As soon as such a target moves behind its companion, thereby itself becoming the farthest target, it virtually ceases to move, while the formerly farther (but now nearer) target begins to change its apparent distance. Although conducted with static displays, Foley's study clearly makes the same prediction as our own development of the far-anchor effect (based upon the specific distance tendency). The only serious difference is that the far-anchor effect (as previously noted) is not conceptualized as a necessarily binocular phenomenon. The relevance of an effective reference parallax distance would be less clear if similar motion-in-depth effects occurred for purely monocular stimuli. However, until such a possibility can be examined, the results are not only consistent with Foley's theory, but represent a direct extension of his experimental observations.

\section{A Possible Application}

Imagine that it is night and a pilot is flying her plane into a busy airspace. In the distance, the lights of a second plane come into view. The pilot visually determines that the other plane is approaching in her general direction at a moderate velocity. Then the lights of a still farther aircraft come into view beyond the nearer plane. If little visual structure besides the two other aircraft were available, it is possible that the pilot's judgments of the speed or direction of the nearer plane (and any subsequent changes in its path) may be affected by the actions of the farthest aircraft. If the farthest plane were also traveling toward her (at a more rapid speed than the nearer plane), our pilot might be subject to any of several alternative perceptual experiences.

First, she might correctly perceive the relative velocities of both approaching planes. However, if the phenomenon examined in the present studies were sufficiently strong, she might perceive that the farthest aircraft was not moving relative to her own position and that the nearer plane was actually moving away! Fortunately, this second possibility is unlikely, because aircraft possess lighting patterns that indicate their direction of travel and (we may presume) there is probably a bias to see at least some motion in such targets. What is more likely (but still potentially dangerous) is the intermediate possibility that both aircraft would be seen as approaching, but their velocities would be subject to misperception. In particular, the nearer plane might be perceived as traveling less rapidly than it really was. That is, the tendency for the farthest object to remain relatively stable in apparent distance (the far-anchor effect) could cause the changing interval between the two planes to be attributed to a slow velocity of the nearer plane. Such an illusion could present a significant hazard if our pilot did not confirm her perceptions with appropriate instrument and/or ground-control information. Although this sort of speculation needs to be confirmed by studies in which both targets are moving simultaneously in depth, the prediction from the faranchor effect seems clear.

\section{REFERENCES}

Aldrich, J. H., \& Nelson, F. D. (1984). Linear probability, logit and probit models. Beverly Hills, CA: Sage Publications.

Ball, K., \& SEKULER, R. (1980). Human vision favors centrifugal motion. Perception, 9, 317-325.

ERKELENS, C. J., \& REGAN, D. (1986). Human ocular vergence movements induced by changing size and disparity. Journal of Physiology, 379, 145-169.

Foley, J. M. (1980). Binocular distance perception. Psychological Review, 87, 411.434 .

FOLEY, J. M. (1985). Binocular distance perception: Egocentric distance tasks. Journal of Experimental Psychology: Human Perception \& Performance, 11, 133-149.

GoGEL, W. C. (1969). The sensing of retinal size. Vision Research, 9, 1079-1094.

GOGEL, W. C. (1972). Scalar perceptions with binocular cues of distance. American Journal of Psychology, 85, 477-497.

GoGEL, W. C. (1977). The metric of visual space. In W. Epstein (Ed.), Stability and constancy in visual perception: Mechanisms and processes (pp. 129-181). New York: Wiley.

GoGEL, W. C. (1984). The role of perceptual interrelations in figural synthesis. In P. Dodwell \& T. Caelli (Eds.), Figural synthesis (pp. 31 82). Hillsdale, NJ: Erlbaum.

Hong, X., \& Regan, D. (1989), Visual field defects for unidirectional and oscillatory motion in depth. Vision Research, 29, 809-819.

Janssen, W. H., Michon, J. A., \& Harvey, L. O. (1976). The perception of lead vehicle movement in darkness. Accident Analysis \& Prevention, 8, 151-166. 
Lappin, J. S. (1990). Perceiving the metric structure of environmental objects from motion, self-motion and stereopsis. In R. Warren \& A. H. Wertheim (Eds.), Perception and control of self-motion. Hillsdale. NJ: Erlbaum.

Mershon, D. H., Granberry-Hager, S., Bartlett, K. \& DeCAMP, B. (1981). Scalar perceptions of distance in a multiobject binocular display. Bulletin of the Psychonomic Society, 18, 249-252.

Mershon, D. H., Lembo, V. L. (1977). Scalar perceptions of distance in simple binocular configurations. American Journal of Psychology, 90, 17-28

Mershon, D. H., Voncannon, M. G., \& Windes, W. R. (1976). Scalar perceptions of distance for a monocularly determined depth interval. Bulletin of the Psychonomic Society, 8, 341-342.

Morrison, J. D., \& Whiteside, T. C. D. (1984). Binocular cues in the perception of distance of a point source of light. Perception, 13, 555-566.

OWENS, D. A., \& LEIBowitZ, H. W. (1980). Accommodation, convergence, and distance perception in low illumination. American Journal of Optometry \& Physiological Optics, 57, 540-550

Perrone, J. A. (1986). Anisotropic responses to motion toward and away from the eye. Perception \& Psychophysics, 39, 1-8.

Regan, D., Erkelens, C. J., \& Collewun, H. (1986). Necessary conditions for the perception of motion in depth. Investigative Ophthalmology \& Visual Science, 27, 584-597.

Rock, I., Auster, M., Schiffman, M., \& Wheeler, D. (1980). Induced movement based on subtraction of motion from the inducing object. Journal of Experimental Psychology: Human Perception \& Performance, 6, 391-403.

\section{NOTES}

1. It is reasonable to speak of a single egocentric reference distance, despite the small changes that occur in the vergence distance of por- tions of the display, because all of the positions of the targets in these studies are indistinguishable when the targets are presented in isolation.

2. In addition to those with obvious fusional difficulties (i.e., breakup of the display into excess targets), there were also observers who reported experiences indicative of related problems (e.g. . seeing a reversed depth relationship of the targets or, during Experiment 2, reporting only lateral motion, rather than motion in depth). Overall, the following additional observers passed the preliminary screening, but were replaced because of difficulty during the experimental presentation(s): Experiment 1, 4 observers (beyond the 60 observers included in the analyses; all from the simultaneous near-far condition); Experiment 2, 79 observers (beyond the 320 in the analyses); Experiment 3, 14 observers (beyond the 48 in the analyses). Not counted in these totals are a few observers who had to be replaced due to procedural errors.

3. The described comparisons appear to involve accepting the null hypothesis and thereby concluding that the perceived distances $\left(D^{\prime}\right)$ of the far target alone and of the far target in combination are necessarily equal. We are, however, only making the more modest statement that there is no reliable evidence of a difference. The design of this type of study makes stronger statements difficult, because the statistical comparisons that one might like to make involve an awkward cross between dependent and independent groups. As a possible alternative, one can legitimately compare $D^{\prime}$ for the near target in the simultaneous presentation (relative to the median $D^{\prime}$ for the same target in the individual presentations) with $D^{\prime}$ for the far target in the simultaneous presentation (relative to the median $D^{\prime}$ for that target in the individual presentations). The difference of the resulting paired values provides a test of whether the near target has shifted by a greater extent than the far target. For the data of Experiment 1 , this comparison shows the expected significance $[t(19)=10.53, p=.0001$, two-tailed $]$.

(Manuscript received August 22, 1991; revision accepted for publication January 25, 1993.) 